\title{
Multi-modal Visualization of Stroke Lesion CT-Imaging
}

\author{
Kurt Schardt*, Robin G. C. Maack*, Dorothee Saur ${ }^{\dagger}$, Hans Hagen*, Gerik Scheuermann ${ }^{\ddagger}$ and Christina Gillmann ${ }^{\ddagger}$ \\ ${ }^{*}$ University of Kaiserslautern, Kaiserslautern, Germany \\ E-mail: kschardt@rhrk.uni-kl.de, maack@rhrk.uni-kl.de, hagen@informatik.uni-kl.de \\ ${ }^{\dagger}$ Leipzig University, Medical Centre, Leipzig, Germany \\ E-mail: dorothee.saur@medizin.uni-leipzig.de \\ ${ }_{\ddagger}^{\ddagger}$ Leipzig University, Leipzig, Germany \\ E-mail: scheuermann@informatik.uni-leipzig.de, gillmann@informatik.uni-leipzig.de
}

\begin{abstract}
Stroke lesions are a result of a sudden cerebrovascular disease that cause a lack of blood supply to the brain. Clinicians aim to localize and quantify brain lesions by utilizing multi-modal CT (Computed Tomography) imaging in order to provide a suitable treatment. In clinical daily routine, neurologists review one modality at a time and a correlation between several modalities is hard to obtain. To better understand the effects of a stroke and identify the origin, we visualize the multi-modal CT data set of a patient by providing a multi-view visualization system. With this visualization we are able to provide a focus and overview of the multi-modal brain lesion imaging available of one patient that allows clinicians to correlate multi-modal stroke imaging. We show the applicability of the presented approach by applying it to real world patient data sets.
\end{abstract}

Index Terms-Medical Visualization, Multi-modal Visualization, Focus and Context Visualization

\section{INTRODUCTION}

Strokes are the second leading cause of disability worldwide [7]. They are caused by a blockage in the supporting vascular system of the brain and can lead to brain lesions. Brain lesions are abnormalities of the brain tissue [2] that can lead to movement, attention, speech and language disorders [1]. The location and size of lesions are an important indicator in order to decide a proper treatment for a patient. Therefore, the occurrence and position of lesions have to be analyzed.

Unfortunately, lesions are hard to determine in an acute stroke imaging. They can form back or grow depending on the patients condition. Therefore, neurologists acquire multiple images of a patients brain during an acute stroke and try to estimate the damage to the brain in order to decide on a proper treatment. Here, original CT scans, angiographies and perfusion maps [4] are utilized, but it is hard to estimate the final lesion based on this imaging. After a healing phase, the lesion is captured by a follow-up image to determine the final damage in the brain. Clinicians aim to correlate this information with the originally captured CT scans. To do so, neurologists review the differently captured modalities independent of each other utilizing the slice-by-slice imaging technique [9], as shown in Section II. Here, medical doctors scroll through a stack of

LEVIA'20: Leipzig Symposium on Visualization in Applications 2020 images to imagine a three-dimensional image of the current patient.

Although there exist a variety of multi-modal visualization approaches for medical purposes, the application of brain stroke lesion imaging is unique and a suitable visualization needs to fulfill specific requirements. As shown in Section III, such a visualization was not achieved so far.

In this paper we present a multi-modal visualization of stroke lesion imaging (see Section IV). The visualization is designed in such a manner, that neurologists can utilize their well-known slice-by-slice reviewing technique to understand each image modality in detail. In addition, we provide an abstract three-dimensional view of all modalities that represent important features of each modality and provides correlation between the different modalities. The views are linked to provide an interactive system.

Therefore, this paper contributes:

- Requirements for the visualization of stroke lesion imaging

- Context view of multi-modal brain lesion imaging

- Detail visualization of single modalities

To show the applicability of the presented approach, we applied our visualization to real world patient data sets (see Section V). Followed by that, we discuss the presented approach in Section VI and conclude our work in Section VII.

\section{APPLICATION AND REQUIREMENTS ANALYSIS}

In this Section we aim to provide further detail about brain lesion imaging and define the requirements for a visualization that allows clinicians to review multi-modal brain lesion imaging.

\section{A. Multi-modal Stroke Imaging}

The following Section describes the different data types and their characteristics utilized in the presented case used for our visualization. The data is provided by the University Hospital Leipzig, that acquired these images during their clinical daily routine. The images are acquired if a patient is consigned into the hospital in suspicion to be affected by lesion. As 

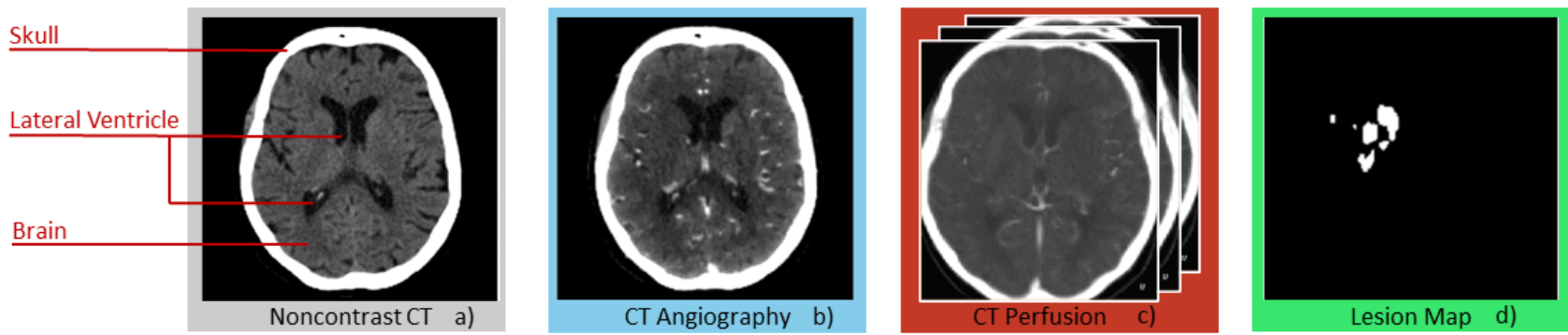

Fig. 1. Multi-modal input of the presented visualization approach showing only one slice per modality. Noncontrast CT, CT Angiography are captured as 3D CT scans, CT perfusion shows a sequence of CT scans and the lesion map holds a mask indicating the identified lesion.

strokes can be hard to determine, several image modalities are acquired to examine the patients brain. Namely, they are Noncontrast CT (NCCT), CT Angiography (CTA), CT Perfusion (CTP), and will be explained in the following. These images form the acute stroke imaging. In addition to this imaging, we obtained a lesion map from our collaborators that summarizes the final lesion in the patients brain. As mentioned before, lesions can grow back or tissue that seemed to recover may die. To understand the relation between the acute stroke imaging and the resulting lesion, our medical collaborators established a project where a follow-up imaging is performed. In this imaging, the final lesion is marked.

Figure 1 shows a slice from each of the modalities, containing Noncontrast CT (NCCT), CT Angiography (CTA), CT Perfusion (CTP) and a Lesion Map. The data sets were linearly coregistered and resliced to the acute CT space using a 12-parameter affine transformation in SPM12 (Statistical parametric mapping). The resulting modalities have a size of $157 \times 189 \times 158$ voxels.

Noncontrast CT: is an ordinary CT scan of the patient's brain, as shown in Figure 1 a). The scan shows the brain of the patient surrounded by the skull. The white matter in the brain can be effected by lesion. The slice number depends on the patients anatomy, but the slice thickness is between 0.8 and $5 \mathrm{~mm}$. The Noncontrast CT gives a first overview of the lesion according to the patients anatomy.

CT Angiography: Angiography is the medical term for the radiological imaging of vessels, mostly blood vessels, by means of diagnostic imaging procedures. After application of a contrast bolus, one volume from the aortic arch to the vertex was acquired [13]. CT Angiography allows to identify blood vessels that can be seen in light color (see Figure $1 \mathrm{~b}$ )). As lesions are a result of blocked vessels, this is an important information for clinicians.

CT Perfusion: CT perfusion is a functional radiological examination method that is used to quantitatively determine cerebral perfusion [3]. During the intravascular injection of a contrast medium containing iodine, several images of the brain are generated in a very short time. CT Perfusion holds 16 images/volume, that capture the propagation of a contrast bolus throughout the time. We use the raw data as input, instead of calculating a parameter map, that shows specific functional parameters. CT perfusion can indicate areas in the brain that are not properly supplied with oxygen. Figure $1 \mathrm{c}$ ) shows the 8th time step of the time-dependent perfusion.

Lesion Map: The lesion data set contains a binary mask indicating voxels that show parts of the lesion. The lesion mask was created by medical doctors utilizing a semi-automated segmentation algorithm [5]. The lesion map was created by a neurologist using a follow-up imaging that contained the final lesion of the patient as shown in Figure $1 \mathrm{~d}$ ). This allows medical doctors to quantify and localize the final lesion of the patient.

\section{B. Requirements for Stroke Lesion Imaging}

In order to provide a suitable visualization of all mentioned modalities in the given medical case, we aim to formulate requirements that need to be fulfilled. Lawonn et al. [12] formulated requirements for multi-modal visualization of medical images in general. We will utilize and adapt these requirements according to long discussions we had with our medical collaborators.

R1: Context visualization to reduce complexity and cognitive load: As mentioned, the currently used slice-by-slice reviewing technique does not allow to review multiple modalities at the same time. Neurologists need to go back and forth or watch modalities side by side in order to obtain an idea which parts of the image are correlated in what manner. Here, important features of all modalities need to be summarized in an overall visualization. We identified the following features as important to visualize based on extensive discussions with our medical collaborators:

- Silhouette of head and brain to examine spatial context

- Vascular system

- Lesion in the brain

- Perfusion information under time changes

R2: Enabling, improving or accelerating decision making: In clinical daily routine, making a decision can be a matter of minutes or seconds. The proposed visualization needs to be usable quickly.

R3: Inclusion of slice-by-slice reviewing: Medical doctors are used to the slice-by-slice reviewing methodology, as they have been trained to interpret medical images in that manner throughout their education. The benefits of this reviewing 
technique (view on the raw data set) need to be preserved in a multi-modal visualization technique.

\section{RELATED WORK}

In the following Section we will summarize related work in the field of multi-modal medical imaging.

Lawonn et al. [12] presented a state of the art analysis that showed the importance of a proper multi-modal visualization in medicine and highlighted the special challenges occurring when dealing with CT imaging. These include different data formats such as scalar field, time-dependent data and segmentation masks. We will use the work of Lawonn as a starting point for our work and expand it to the presented use case.

Gillmann and Salz [10] showed how a suitable visualization of multi-modal medical image data helps improving the communication for medical doctors. Their approach is designed for visualizing images of a patients lung, which needs to be adjusted to the given use case.

Frank et al. [8] as well as Nadeem and Kaufmann [14] provided a multi-modal visualization tool for brain imaging, that allows to map information retrieved by different modalities onto a model of the brain or a hemisphere representing the brain surface. Although this allows a quick visualization of surface properties of the brain it does not allow examination of properties within the brain.

Tolxdorff et al. [17] show several articles on visualization of cortical anatomy (MRI), brain activity (fMRI) and nerve tracts (DTI). Eichelbaum et al. [6] shows a similar approach and made it freely available as the openWalnut tool. Although they were able to merge different modalities into one visualization their approach is not suitable for lesions as it mainly contains structural information.

Rieder et al. [16] presented an interactive method for visualizing multi-modal volume data in neurosurgical therapy planning. These methods allow surgeons to examine multimodal volumes and find a strategy to remove a tumor and minimize damage to other tissue. Unfortunately, it does not allow to include time-dependent data as well.

Peled et al. [15] presented a tool called Multi-Modality Visualization Tool, which is designed for the visualization and exploration of neuroimaging data. It shows purely around the spatial distribution of a functional network and its temporal evolution.

Gillmann et al. [11] presented a visualization approach that shows the uncertainty contained in a computed tomography scan of a brain lesion. In their work, they provided uncertaintyaware paradigms to visually indicate the variability of the resulting stroke.

In contrast to the presented approaches, this work will focus on a multi-modal visualization that helps clinicians review multi-modal stroke imaging data sets utilizing a suitable extension of the state of the art slice-by-slice reviewing methodology.

\section{Methods}

In this Section we will present a multi-modal brain lesion visualization that allows clinicians to review multiple modalities

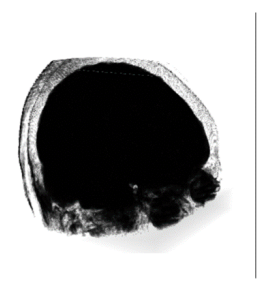

a)

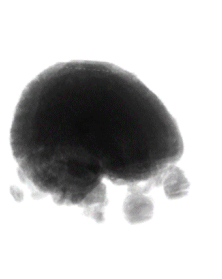

b)

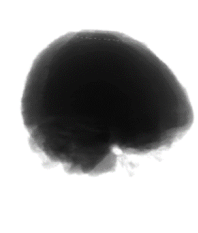

c)
Fig. 2. Creation of brain mask, showing different steps that need to be accomplished. a) Original scan is shown using an opacity transfer function. Image b) shows the result after using the weight function and c) is the result of the recursive function and the final brain mask.

in brain lesion imaging according to the defined requirements in Section II. To achieve this, our approach consists of multiple steps:

- Pre-processing of data sets

- Creation of Overview and detail view

These steps will be explained in the following. In order to understand the described algorithms, we require a set of definitions. Let $I$ be an image, then the following definitions can be applied:

- $I(v)$, return the voxel value at the selected voxel $v=$ $(x, y, z)$

- $\operatorname{dim}(I)$, returns the three dimensions of $I$, namely $X, Y, Z$

\section{A. Pre-processing of data sets}

As mentioned before, we aim to derive an overview of all modalities involved in brain lesion imaging. Therefore, we need to extract important structures from all modalities, Since the CT images do not only contain the brain we need to identify the pixels that solely show parts of the patient's head. This can be easily achieved by using a threshold that excludes air and noise around the patient's head. However, threshold is not sufficient to separate further structures within the patient head. Here, we require a more complex segmentation algorithm, which will be described in the following.

1) Segmentation of Brain: To segment the components included in the available image data, we first require a segmentation of the patients brain. We will refer to this segmentation as the brain mask. Since the brain mask has to be adapted to the data sets, the mask will be segmented from the noncontrast CT. First, we can exclude bones and other unimportant components by using the Hounsfield Unit (HU), which is a typical unit of measurement for CT scans. In our examples, we only keep voxels with the scalar value between 0 and 35 .

This area results from the Houndsfield table and is denser than water but not as dense as bone. Figure 2 a) shows all voxels that are selected when applying the selected threshold. 
As can be seen, the patients brain and skin remain after applying the threshold.

To remove unnecessary components like skin we created a weight function, and we used the same range from $r_{\min }=0$ to $r_{\max }=35$ as the opacity function.

The function examines a voxel and its neighbors (26 in the three-dimensional case). The number of neighbors can be selected as desired. We conducted a variety of tests, but we determined we solely need to consider the direct neighborhood, as the fragments were removed sufficiently well. All neighbors, with a scalar value in the designed limits, are counted and divided by total number of neighbor voxels. If this ratio is above an arbitrarily defined threshold $\omega$, the voxel is kept. In our case we have set the threshold to $30 \%$.

The weighting is used to remove rough fragments outside the brain. Of course, this weighting also affects the brain that has already been identified. Especially at the edges of the brain voxels are removed. To avoid heavy wear and tear, the parameters of the sieve are kept small. To remove most of the fragments, the weighting function is repeated. Here we have realized throughout several experiments by testing, that 3 iterations $t$ give a good result in all considered cases.

The functions input 1 are the scalar ranges from minimum $r_{\min }$ to maximum $r_{\max }$, and the NCCT scan. The output is a new image $N C C T^{*}$ solely containing the brain and larger remaining artifacts. The result resembles the image in Figure $2 \mathrm{~b}$ ). Here, we see, that most structures that do not belong to the brain have been removed.

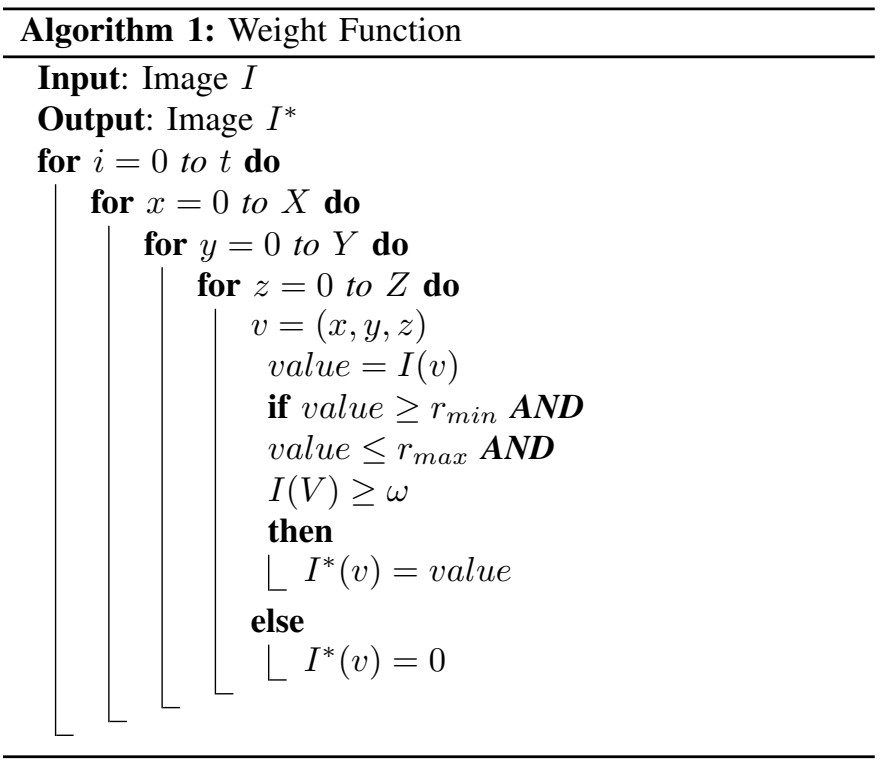

The function separates the brain from the remaining tissue. If the parameters are chosen well as described, the eyeballs are no longer connected to the brain now and three connected components are left (two eyeballs and the brain). If the brain is not fully separated the eyeballs might remain in the masks, which does not affect the following steps.

To remove excess tissue and the eyeballs, another algorithm is applied to the result of the weighting function. As medical

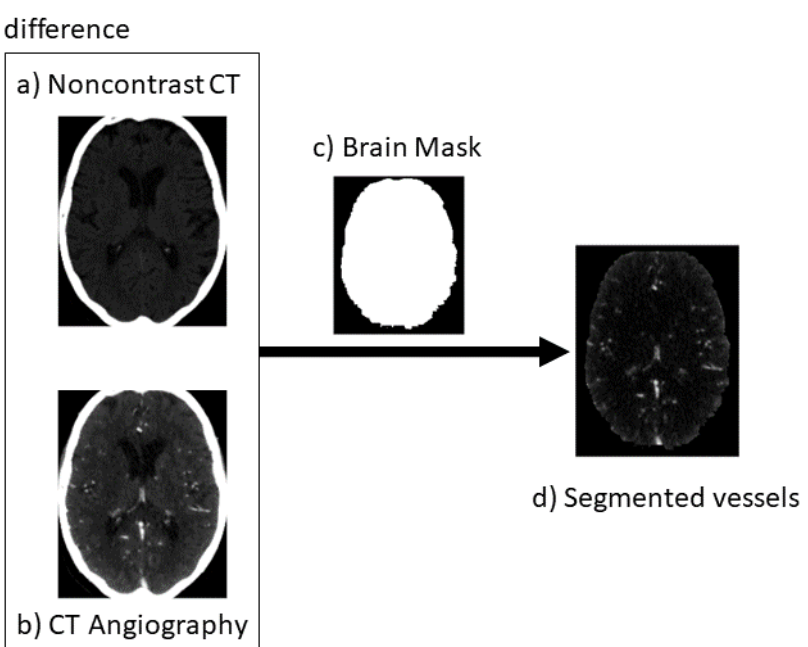

Fig. 3. In the first step the difference of single voxels between the noncontrast CT (a) and the angiography (b) is calculated. Then a mask is placed over it (c). As a result, we get the segmented vessels (d) using an Opacity Transfer Function.

doctors aim to center the patient in the captured brain, we can assume that the center pixel of the captured image is part of the patients brain. Starting from this pixel, we can apply a flood fill algorithm that successively visits all neighbor pixels from the starting pixels. If these neighbor pixels are contained in the previously achieved map, we can count the pixels in the brain. All visited voxels are stored in the final mask $I_{\text {Mask }}$. A summary of the entire mask generation procedure can be found in Algorithm 2. Input is the mask oldImage created with the weight function and a starting point in the brain. With the given CT scans, the brain is always exactly in the middle and the center is always guaranteed to be a part of the brain.

The output of the function is a mask that contains only the brain of the patient utilizing boolean values, which looks like the image in Figure $2 \mathrm{c}$ ).

2) Segmentation of Vascular Structures: A further important structure when analyzing brain lesions are the vessels present in the patients brain. As the lesion is a result of a blocked vessel, we need to highlight these structures in the aimed overview.

The vessel segmentation algorithm considers the difference between the noncontrast CT for angiography and perfusion. This is important as the angiography aims to highlight the vessels and shows if there are vessels that are not properly supplied by blood. The contrast changes in vessels can be segmented when observing the angiography. As shown in Figure 3 the noncontrast CT (a) and the CT angiography (b) are used to calculate the absolute difference of the scalar values of all voxels. Now the brain mask (c) is used as a stencil map removing voxels outside the brain.

After the procedure is done, we obtain a mask for vascular structures. 


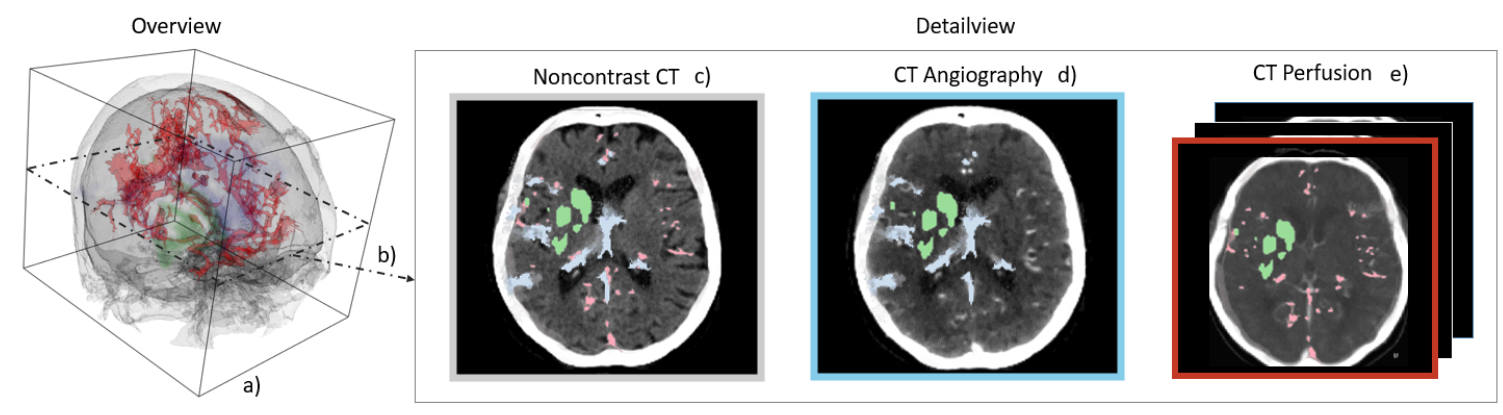

Fig. 4. Overview and detail view of multi-modal brain lesion imaging. Overview of all modalities in the given data set (a). A layer in the overview shows the height of the 3D image at which the current slides of the detail view are located b). The detail view shows the images of the noncontrast CT (c), the CT angiography (d) and the CT Perfusion (e). In each slice the lesion is marked with green and this can also faded out.

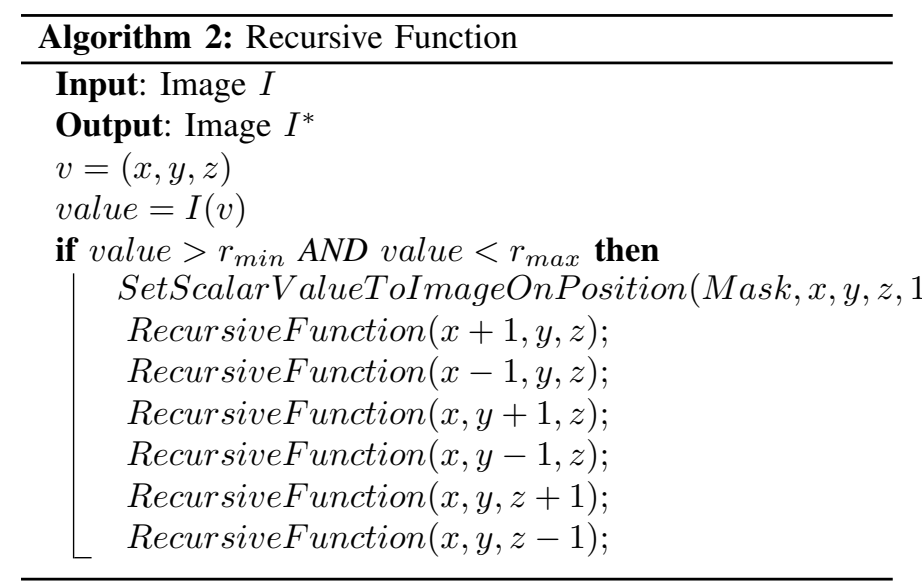

\section{B. Visualization}

After the pre-processing step that extracts all important structures in the brain, we are able to create a multi-view system that allows clinicians to examine multi-modal brain lesion data. The visualization consists of two parts:

\section{- Overview}

- Detail view

The different views and their interaction are shown in Figure 4. The overview shows the 3D images of all parts of the data set which makes it easier for clinicians to identify important features in all data sets. We kept a consistent color scheme throughout this process which is based on the background colors of each modality in Figure 1. This allows the user to identify which feature originates from which modality. By combining the 2D slides in the detail view and the 3D view in the overview, the spatial view is improved without disturbing the previous workflow of the medical professionals.

1) Overview: The goal of the presented overview is to show features that are important across all modalities in the presented data set. As shown in Section II-B our medical collaborators clearly expressed features that are interesting for

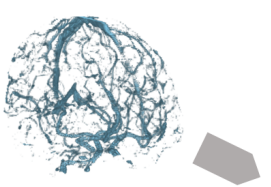

a)

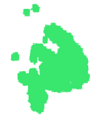

c)

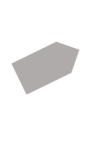

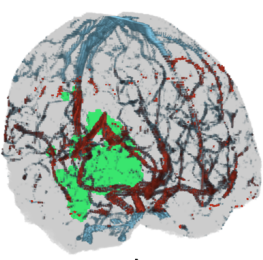

e)

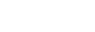

d)

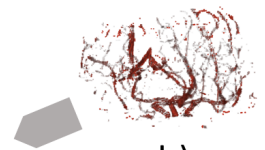

b)

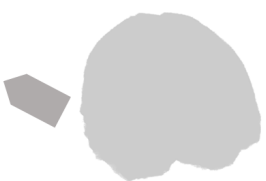

d)
Fig. 5. Overview of components in the overview visualization. Extracted surface of the segmented patients brain blood system from the CT Angiography scan (a). Volume rendering of segmented perfusion (one time-step) (b). Volume rendering of the segmented lesion (c). Extracted surface from the segmented patients brain (d) using noncontrast CT. Merged visualization (e).

the overview. In order to achieve this, we extracted important features from the multi-modal data set and combined them to a context visualization.

As shown in Figure 5 the overview consists of 4 components. The brain of the patient is displayed by an isosurface. We chose this visualization as we solely need a silhouette of the brain to show spatial context. The silhouette is shown transparent to provide a look into the brain. Within the brain, we show the extracted vascular system as a volume rendering. Here, we first show the perfusion data in blue. As an overlay we show the vessels extracted from the angiography to highlight the vessels that are currently examined in the data set. At last, we provide a volume rendering of the extracted lesion of the patient.

A further function of the overview is that all time-steps of the perfusion can be run through in one animation. This helps to understand the blood flow and its direction. Figure 6 shows how the blood flows during the captured perfusion scan. Users can easily determine which parts of the vascular system 
are supplied in the current time step and how the perfusion distributes along the vascular system.

In addition, we allow including or exclude all components that are visible in each of the views to allow clinicians to focus on aspects in the data sets that they are interested in

Overall, the overview display enables medical practitioners to view a multi-modal stroke data set in a single presentation. It reduces cognitive overload such that only the most important features of all data sets are retained.

2) Detail view: The detail view displays the original data records. This was an explicit wish from our medical collaborators as they needed a visualization where they can review the raw data sets. Clinicians can select a specific modality and review them by scrolling through the single slice. Since the Clinicians scrolls through the CT scans layer by layer, we provide this functionality using a slider. The overview and detail view are closely linked, allowing medical doctors to switch freely between them. Here, slices are inserted into the overview to indicate which modality is currently reviewed in which slice.

Furthermore, the perfusion can be animated, displaying all time-steps in sequence. In figure 4 the detail views side is shown. CT scans are usually captured as scalar fields; therefore, they do not include color information. The medical professionals analyze the image by looking for homogeneously bright areas that are visible through the contrast medium. This way they can look at the vessels in the angiography, the blood flow in perfusion, and the damage in the lesion map. Since there are no smooth and visible edges, they must roughly estimate the area of interest.

To enhance the slice by slice view further and keep a correlation to the remaining modalities, we allow an inlay of the previously extracted structures from all modalities. Figure 4 shows three slice by slice views wherein each view, the remaining modalities are presented as an overlay. Here, clinicians can make direct correlations between the lesion and the currently reviewed modality.

This allows medical professionals to view their data in the usual way and to go through their daily work processes.

\section{Results}

In the following we apply our approach to two different real-world data sets that we obtained from our medical collaborators. The images were acquired at the University Hospital in Leipzig.

\section{A. Example 1}

Figure 5 shows the resulting visualization of the presented method, when applying it to the first data set. Figure 5 a) shows the overview containing isosurfaces and volume rendering components. The head and brain of the patient are shown as transparent isosurfaces (grey). The vascular system is shown as a transparent blue isosurface. Voxels that are indicated as lesion in the lesion map are shown using green volume rendering. The perfusion information of the 7 th time-step in the CT perfusion scan is shown by a red volume rendering.
Neurologists can scroll through the volume and depict a slice that they are aiming to review in detail (see Figure $5 \mathrm{~b}$, c and d). This allows an overview of the entire multi-modal data set.

Figure 5 b), c) and d) also holds the extended slice-by-slice view but contains the CT angiography scan, the CT perfusion scans and the CT lesion map respectively. This directly helps medical doctors in correlating the different modalities in the data set. Please note that the perfusion information is changing throughout time as it presents a time-dependent data set. The visualization is based on the slice-by-slice reviewing technique utilized in clinical daily routine. Thus, neurologists can directly use the presented visualization without requiring extensive training.

\section{B. Example 2}

With the methods mentioned above, all individual parts of the brain were automatically segmented. In Figure 7 we can see the individual components of the brain (a) in gray, the angiography (b) in blue and the lesion (c) in green. The perfusion is not listed because of missing data. Also, the assembly of all components is visible in image (d), the way they look like in the overview.

Figure 8 shows the angiography at all stages. On the one hand, the angiography recording is shown without manipulation of the data set (a). This is also shown in the detailed view such that physicians can view their untreated slides. In the middle the treated data slide is shown (b). From this data set the $3 \mathrm{D}$ view on the right side (c) is created.

Figure 9 shows the lesion once in the 2D view (a) and once in the $3 \mathrm{D}$ view (b). The $2 \mathrm{D}$ view is displaying the raw data as a slice-by-slice visualization.

As one can see in the overview Figure 7, the lesion is located in an area where the vascular systems seem to vanish. Here, medical doctors can directly identify the potential vessels that can contain a blockage. The remaining vascular system seems healthy and all important vessels are present and contain blood. The area around the lesion seems fuzzy which indicates a blocked vessel.

\section{DISCUSSION}

In this Section we aim to discuss the formulated requirements, shows how clinicians rated our visualization and provide a bridge to the visualization community in general.

\section{A. Improvements in multi-modal brain lesion imaging}

The presented visualization holds the defined requirements formulated in Section II-B. The overview allows clinicians to examine important features from all modalities, namely the head of the patient, the brain silhouette, the vessels in the brain (active and inactive) and the final lesion (R1).

In addition to that, the overview provides clinicians with the ability to directly focus on important aspects of the current patient and directly decide which modality needs to be examined in further detail (R2).

At last, we allowed the use of the well-established slice-byslice reviewing procedure in medicine. Although this approach 


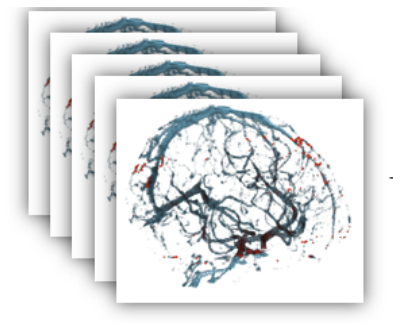

Time-steps 0 - 4

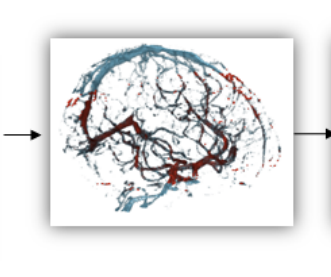

Time-steps 5 - 7

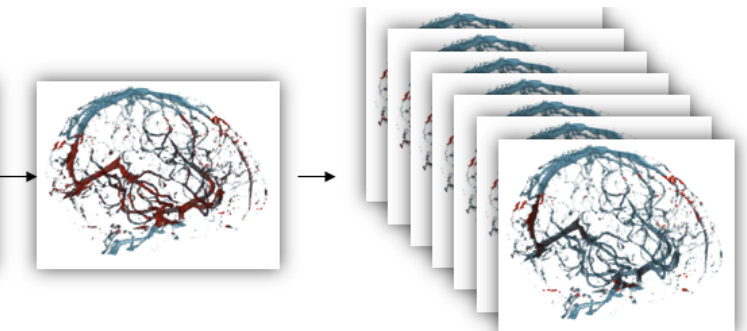

Time-steps 8 - 14

Fig. 6. Timesteps of perfusion. The time-steps 5, 6 and 7 are displayed separately. They show how the blood (indicated by red) flows through the blue colored vessels.

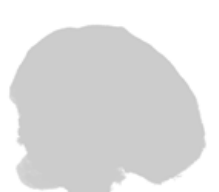

a)

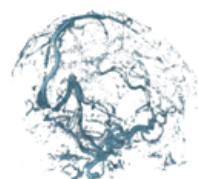

b)

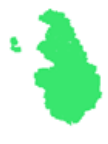

c)

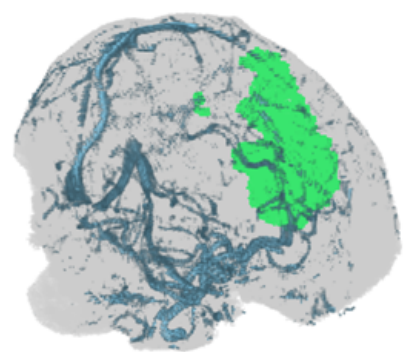

d)

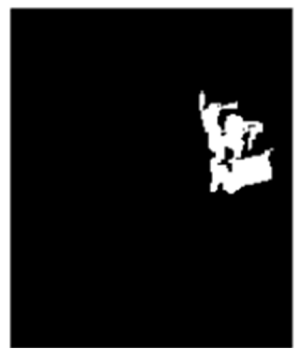

a)

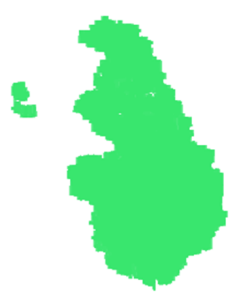

b)

Fig. 7. This is another data set that includes the native brain (a), angiography (b) and lesion (c). On the right side the composition of the individual parts can be seen (d).

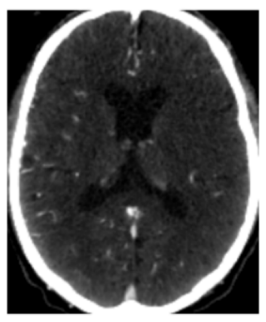

a)

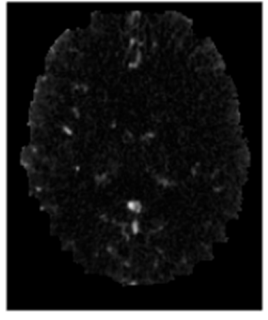

b)

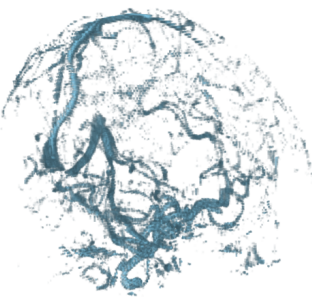

c)

Fig. 8. On the left side you can see a slice of the angiography before the data was prepared by our presented methods, (a) as it would look like in the detail view. The same slice is displayed in the middle after processing the data (b). Here, unnecessary components such as bones have already been extracted. On the right side a slice of the angiography is shown in a 3D view (b), as it would look like in the overview.

has its drawbacks, clinicians are used to this method and request it to be included in any novel visualization approach. We extended this methodology by providing an overlay of important aspects extracted from the remaining image modalities that can be switched on and off as required.

Overall, the visualization can help neurologists correlate acute stroke data sets with the resulting lesion in the patients brain.

\section{B. Expert feedback}

We presented the developed visualization to our small team of medical collaborators in an informal user study. Here, we introduced the visual concepts and let our medical collaborators explore the system by their own. During this evaluation we collected expert feedback, that will be summarized below.

Our medical collaborators were very satisfied by the overview. They claimed that this view would not be sufficient to achieve a diagnosis by itself, but the view is able to provide a broad overview over all modalities. The clinicians especially liked the 3D view as they are now able to find spatial correlations on vessels that are blocked and the resulting lesion in the brain. In addition, our collaborators obtained a direct visual hint which modality needs to be examined further.

Our collaborators were also very pleased that the sliceby-slice reviewing was included in our methodology. They 
claimed that this is in some cases the only way to make a final decision. They liked the interactivity of the approach and the correlation between overview and detail view by the provided interaction.

\section{Impact to VIS community}

As shown by Lawonn et al. [12], multi-modal visualization is an important problem regarding medical image data. In the presented work we showed a solution for a specific use case in medicine. Nevertheless, we identified a set of important visual requirements that are necessary to provide a successful visualization.

Besides medicine, further applications are concerned with multi-modal data sets, such as mechanical engineering. These applications can also learn from the presented way. We showed that a close collaboration is important to provide useful visualization in a given context and would like to encourage other researchers to proceed as presented.

\section{CONCLUSiOns AND Future WORK}

We presented a multi-modal interactive $3 \mathrm{D}$ visualization of the brain containing an overview and a detail view. The overview merges important features of all modalities contained in the data set whereas the detail view shows a depicted modality with segmented elements of the remaining modalities. The usability of the presented approach was shown by applying it to a real world data set acquired in clinical daily routine. We showed how this visualization can help neurologists to correlate acute stroke imaging to the remaining lesion in the patients brain.

In future work, we aim to provide an open access tool containing the developed visualization for neurology research. In addition, we aim to perform an extensive study on the correlation between acute stroke imaging and the resulting lesion.

\section{REFERENCES}

[1] R. Adolphs. Human lesion studies in the 21st century. Neuron, 90(6): $1151-1153,2016$

[2] J. Alstott, M. Breakspear, P. Hagmann, L. Cammoun, and O. Sporns. Modeling the impact of lesions in the human brain. PLOS Computational Biology, 5(6):1-12, 062009.

[3] A. Bivard, C. Levi, N. Spratt, and M. Parsons. Perfusion ct in acute stroke: A comprehensive analysis of infarct and penumbra. Radiology, 267(2):543-550, 2013. PMID: 23264345.

[4] V. D. Calhoun and J. Sui. Multimodal fusion of brain imaging data: a key to finding the missing link (s) in complex mental illness. Biological psychiatry: cognitive neuroscience and neuroimaging, 1(3):230-244, 2016.

[5] B. de Haan, P. Clas, H. Juenger, M. Wilke, and H.-O. Karnath. Fast semi-automated lesion demarcation in stroke. NeuroImage: Clinical, 9:69- 74, 2015.

[6] S. Eichelbaum, M. Goldau, S. Philips, A. Reichenbach, R. Schurade, and A. Wiebel. Openwalnut: A new tool for multi-modal visualization of the human brain. Proceedings of the EG VCBM, 2010.

[7] V. L. Feigin, B. Norrving, and G. A. Mensah. Global burden of stroke. Circulation Research, 120(3):439-448, 2017.

[8] R. Frank, H. Damasio, and T. Grabowski. Brainvox: An interactive, multimodal visualization and analysis system for neuroanatomical imaging. NeuroImage, 5(1):13 - 30, 1997.
[9] Gillmann. Intuitive Error Space Exploration of Medical Image Data in Clinical Daily Routine. In B. Kozlikova, T. Schreck, and T. Wischgoll, editors, EuroVis 2017 - Short Papers. The Eurographics Association, 2017.

[10] C. Gillmann and P. Salz. Improving visual communication for eit-based lung research. In 2014 IEEE Pacific Visualization Symposium, pages 291-295, March 2014.

[11] C. Gillmann, D. Saur, T. Wischgoll, K.-T. Hoffmann, H. Hagen, R. Maciejewski, and G. Scheuermann. Uncertainty-aware Brain Lesion Analysis, 2020.

[12] K. Lawonn, N. Smit, K. Bühler, and B. Preim. A Survey on Multimodal Medical Data Visualization. Computer Graphics Forum, 2018.

[13] M. Matsumoto, N. Kodama, Y. Endo, J. Sakuma, K. Suzuki, T. Sasaki, K. Murakami, K. Suzuki, T. Katakura, and F. Shishido. Dynamic 3dct angiography. American Journal of Neuroradiology, 28(2):299-304, 2007.

[14] S. Nadeem and A. Kaufman. Multimodal brain visualization. In B. Gimi and A. Krol, editors, Medical Imaging 2016: Biomedical Applications in Molecular, Structural, and Functional Imaging, volume 9788, pages 503 - 510. International Society for Optics and Photonics, SPIE, 2016.

[15] O. Noam Peled and ofek schechner. pelednoam/mmvt: First beta version, mar 2017.

[16] C. Rieder, F. Ritter, M. Raspe, and H.-O. Peitgen. Interactive Visualization of Multimodal Volume Data for Neurosurgical Tumor Treatment. Computer Graphics Forum, 2008.

[17] T. Tolxdorff, T. M. Deserno, H. Handels, A. K. Maier, K. H. MaierHein, and C. Palm, editors. Bildverarbeitung für die Medizin 2020 Algorithmen - Systeme - Anwendungen. Proceedings des Workshops vom 15. bis 17. März, 2020 in Berlin, Informatik Aktuell. Springer, 2020. 